\title{
Transcutaneous Electrical Nerve Stimulation of PC5 and PC6 Acupoints Increases Sympathovagal Balance but Not Oxidative Stress in Healthy Subjects: A Randomized Clinical Trial
}

\author{
Fernando Gomes de Jesus ${ }^{1,2}$, Alice Pereira Duque ${ }^{1,2}$, Carole Sant'ana Massolar ${ }^{1}$, Giselle Pinto de Faria Lopes ${ }^{1}$, \\ Ana Carolina de Azevedo Carvalho ${ }^{3}$, Mauro Felippe Felix Mediano ${ }^{2,4}$, Luiz Fernando Rodrigues Junior ${ }^{1,2, *}$ \\ ${ }^{1}$ Department of Physiological Sciences, Biomedical Institute, Federal University of the State of Rio de Janeiro, Rio de Janeiro, RJ, Brazil \\ ${ }^{2}$ Education and Research Department, National Institute of Cardiology, Rio de Janeiro, RJ, Brazil \\ ${ }^{3}$ Federal Institute of Education, Science, and Technology of Rio de Janeiro, Rio de Janeiro, RJ, Brazil \\ ${ }^{4}$ Evandro Chagas National Institute of Infectious Diseases, Oswaldo Cruz Foundation, Rio de Janeiro, RJ, Brazil
}

Received February 6, 2021

Revised July 9, 2021

Accepted July 22, 2021

Correspondence to Luiz Fernando Rodrigues Junior Department of Physiological Sciences, Biomedical Institute, Federal University of the State of Rio de Janeiro, Rio de Janeiro, RJ, Brazil

E-mail luiz.junior@unirio.br
Background: Transcutaneous electrical acupoint stimulation (TEAS) improves autonomic balance and reduces oxidative stress in subjects with chronic diseases, that decreases the risk of low-grade chronic inflammatory diseases, including cardiovascular diseases. However, these beneficial effects have never been demonstrated in healthy subjects. Objectives: To evaluate the acute effects of TEAS on autonomic balance and oxidative stress of healthy subjects.

Methods: A randomized clinical trial was conducted with male healthy subjects (1830 years old), randomly allocated to control (no intervention; $n=14$ ), placebo (placebo intervention; $n=14$ ) and TEAS group (at PC5 and PC6 acupoints; $n=13$ ). The protocol consisted of accommodation (20 min), intervention (40 min), and recovery (30 $\mathrm{min}$ ) periods. The acute effects of TEAS on hemodynamics were studied through measurements of heart rate, blood pressure and double product; on the autonomic nervous system by assessing heart rate variability; and on oxidative stress by quantifying reactive oxygen species in saliva samples, collected at the end of each period.

Results: TEAS increased heart rate and double-product compared to control and placebo groups $(p<0.01)$. Moreover, TEAS increased sympathetic and reduced parasympathetic tonus, increasing the sympathovagal balance compared to the control and placebo groups. However, TEAS exerted no effect on oxidative stress in saliva samples.

Conclusion: In healthy subjects, TEAS at PC5 and PC6 acupoints acutely improved autonomic balance, increasing sympathetic and reducing parasympathetic tonus, reflecting little improvement on hemodynamic responses. Whether it could be used as a cardioprotective strategy remains uncertain since it exerted no effect on oxidative stress.

Keywords: Transcutaneous electric nerve stimulation, Acupoints, Autonomic nervous system, Oxidative stress

\section{INTRODUCTION}

Cardiovascular diseases (CVDs) are the main cause of deaths worldwide, responsible for approximately 17.3 million deaths per year, with estimates of more than 20.3 million deaths per year by 2030 [1]. It is estimated that the global cost for CVDs prevention and treatment will be U\$ 47 trillion over the next 25 years [1]. Moreover, their impact have dramatically increased in the less developed countries.
For example, in the last two decades, premature deaths from CVDs reached $42 \%$ of all deaths registered in low-income countries and $80 \%$ of deaths caused by CVDs globally were registered in low or middle-income countries [2-4].

From a pathophysiological spectrum, several CVDs are associated with autonomic nervous system (ANS) dysfunction, with sympathetic hyperactivation and/or inhibition of parasympathetic modulation, being a consequence or even the cause of such disorders [5]. Furthermore, individuals 
without CVDs, considered healthy, may suffer from autonomic disorders associated with some conditions, which can induce or facilitate the development of CVDs in a long-term [6].

Therefore, non-pharmacological and non-invasive approaches that enhance ANS control of heart could benefit healthy subjects, acting as prophylactic therapy. One such emerging strategy is electroacupuncture that, when applied at the Neiguan (PC6) and Jianshi (PC5) points, improves left ventricular contractility, mean arterial pressure, cardiac output, and vascular blood pressure responses through sympathetic system activation [7-9]. Also, transcutaneous electrical acupoint stimulation (TEAS), a noninvasive method for prescribing electroacupuncture [10,11], at PC6 acupoint modulates autonomic balance in obese people [12]. Even more, it was already demonstrated in animal models that both acupuncture [13], electroacupuncture [14] and TEAS [15] can reduce oxidative stress, which is strongly associated with development of low-grade chronic inflammatory diseases, including CVDs. However, both effects have never been demonstrated in healthy subjects.

Thus, the present study aims to evaluate the acute effects of transcutaneous electrostimulation at PC5 (Jianshi) and PC6 (Neiguan) acupuncture points on the autonomic balance and oxidative stress of healthy individuals.

\section{MATERIALS AND METHODS}

\section{Study design}

The present study was a single-blind, placebo-controlled, clinical trial conducted from January 2016 to March 2017. Healthy volunteers were recruited in the Biomedical Institute at Federal University of the State of Rio de Janeiro, Rio de Janeiro, Brazil. Male healthy subjects with age $\geq 18$ and $\leq 30$ years old and that self-reported no acute or chronic disease at the time of enrollment were included in the study. Exclusion criteria were history of implantable heart pacemakers or cardioverter defibrillators, use of auditory devices, brain injury, status epilepticus, and tetanus. The total sample size of 33 subjects size (11 per group; calculated based on data obtained from the literature using the mean and the standard deviation of reactive oxygen species (ROS), considering alpha error of 0.05 and beta error of 0.05 [16]) was expanded by $30 \%$ considering possible losses, achieving 14 subjects per group. The included volunteers received a numeric code and were simple random allocated (Excel software, Microsoft, USA) in three distinct groups: control, placebo and intervention group submitted to TEAS (TEAS group).

\section{Intervention}

A single TEAS session was performed by an experienced physiotherapist at an intensity of $0.8-1.9 \mathrm{~mA}$ and a frequency of 5-30 Hz (Neurodyn Tens Port; Ibramed Series 92875; ANVISA registry: 10360310012) [17]. The electrodes were placed bilaterally at the PC5 (Jianshi) and PC6 (Neiguan) acupuncture points, which are part of the pericardium meridian, located in the anterior region of the forearm near the wrist [18]. The pericardium meridian is postulated to regulate cardiac parameters such as heart rate (HR) [19]. The intervention was carried out in a quiet room with a controlled temperature $\left(23^{\circ} \mathrm{C}\right)$. The patients remained in the supine position during the whole procedure, which consisted of three periods: baseline ( 20 minutes to allow for HR and blood pressure accommodation), intervention (40 minutes), and recovery (30 minutes to allow for HR and blood pressure recovery). The control and placebo groups performed the protocol following the same conditions. The placebo group had the electrodes positioned but without electrostimulation whereas in the control group no electrode was positioned and no electrostimulation was performed.

\section{Measurements}

Information was collected on individual assessment sheets filled out by the same researcher previously trained to perform all the procedures of the study, including the questionnaires and anthropometric and blood pressure measurements. The following relevant baseline data were collected: age, gender, previous disease and behavioral factors (physical inactivity, smoking, and alcohol consumption). Primary outcomes were heart rate variability (HRV) and ROS. secondary outcomes were hemodynamic variables.

During the experimental protocol, the arterial blood pressure and HR were measured every 10 minutes from the beginning of the study protocol (baseline period) until the end of the recovery period. The systolic blood pressure (SBP), diastolic blood pressure (DBP), mean arterial pressure (MAP) and double product (DP, a product of $\mathrm{HR}$ and $\mathrm{SBP}$ ) were registered (Digital Sphygmomanometer MA100, G-Tech, Onbo Electronic, Shenzhen Co, China). The delta for systolic blood pressure $(\triangle \mathrm{SBP})$, diastolic blood pressure $(\triangle \mathrm{DBP})$, mean arterial pressure $(\triangle \mathrm{MAP})$ and $\mathrm{DP}(\triangle \mathrm{DP})$ were calculated considering the end of the baseline period (20 minutes of protocol) as the reference timepoint.

In order to guarantee a blinded evaluation of the studied parameters, two professionals were involved in data collection (physiotherapist and principal investigator). The physiotherapist was blinded while the principal investigator was aware of the participant's allocation. The initial evaluations were performed by the blinded physiotherapist that left the room after the baseline measurement procedures. At this time, the principal researcher entered into the room to insert the electrodes, turn on the electrostimulator (except 
for control group), and cover the volunteer (only leaving the head exposed) and the electrostimulator with a light blanket. Afterwards, the physiotherapist returned to the room and followed the patient during the remaining study procedures. The evaluations of the HRV and ROS parameters were also blinded for the investigators.

\section{Autonomic function analysis}

Autonomic function was studied using HRV analysis. The RR interval was continuously recorded throughout the experiment using a cardiofrequencimeter (RS800; Polar, USA) and subsequently used to evaluate the HRV using appropriate software (Kubios v. 2.2; UEF, Finland). The following timedomain HRV parameters were evaluated: mean of all normal RR interval (mean RR), standard deviation of normal
RR interval (SDNN), number of consecutive normal RR interval differences greater than $50 \mathrm{~ms}$ (NN50), percentage of normal RR intervals that differed by more than $50 \mathrm{~ms}$ from the adjacent interval (pNN50), root-mean-square of successive differences (RMSSD). Integration of the successive HR bands was classified concerning the frequency domain as follows: very low frequency component (VLF; 0.003$0.04 \mathrm{~Hz}$ ), low frequency component (LF; 0.04-0.15 Hz), high frequency component (HF; 0.15-0.4 Hz). The ratio of LF to HF (LF/HF) was used to calculate the sympathovagal index. The normalized power of the LF and HF components was calculated in standard units (nu).

\section{Oxidative stress analysis}

Saliva samples were collected at the final minute of

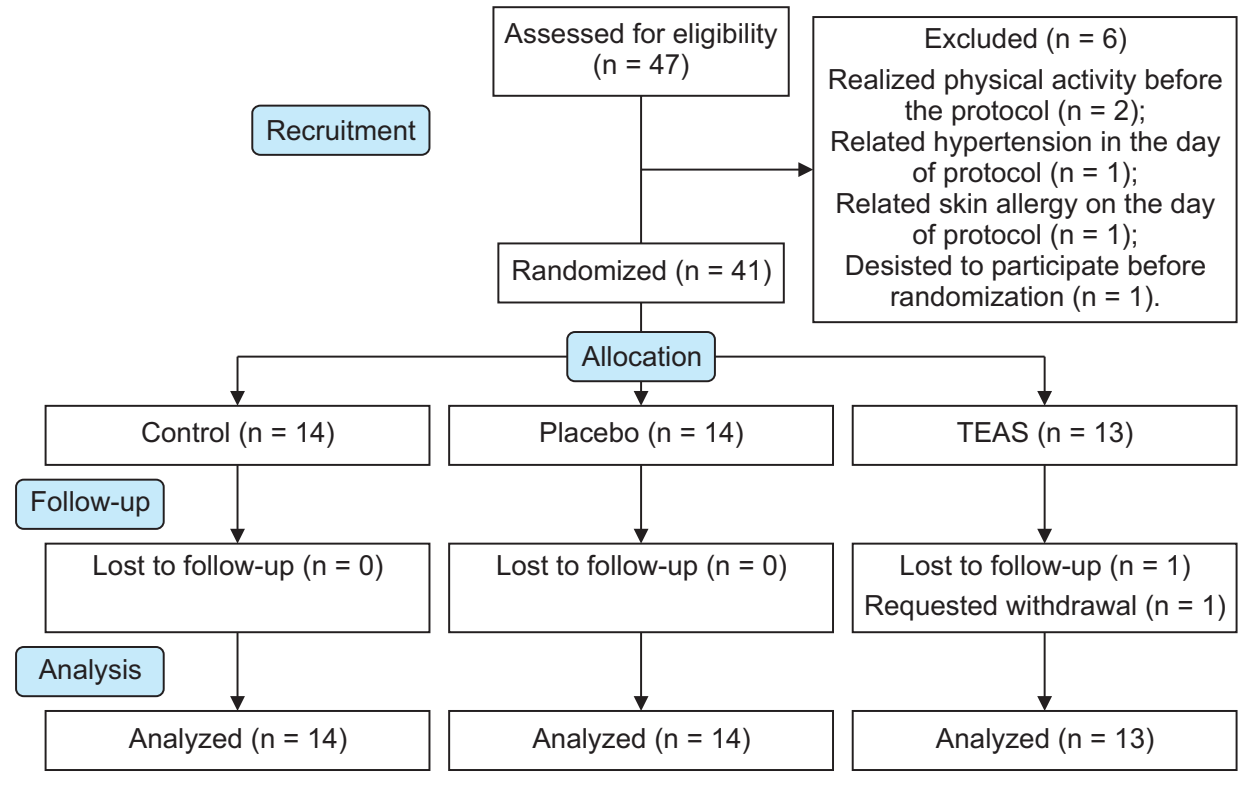

Fig. 1. Study flow diagram.

Table 1. Baseline characteristics of volunteers

\begin{tabular}{|c|c|c|c|c|}
\hline Variable & $\begin{array}{c}\text { Control }(\mathrm{N}=14) \\
\mathrm{N}(\%) \text { or mean } \pm \mathrm{SE}\end{array}$ & $\begin{array}{c}\text { Placebo }(\mathrm{N}=14) \\
\mathrm{N}(\%) \text { or mean } \pm \mathrm{SE}\end{array}$ & $\begin{array}{c}\text { TEAS }(\mathrm{N}=13) \\
\mathrm{N}(\%) \text { or mean } \pm \mathrm{SE}\end{array}$ & $\begin{array}{c}\text { Total }(\mathrm{N}=41) \\
\mathrm{N}(\%) \text { or mean } \pm \mathrm{SE}\end{array}$ \\
\hline Age (years old) & $21.1 \pm 0.6$ & $21.1 \pm 0.6$ & $20.9 \pm 0.6$ & $21.1 \pm 0.3$ \\
\hline Weight (kg) & $82.4 \pm 3.8$ & $70.8 \pm 2.8$ & $70.9 \pm 5.0$ & $76.0 \pm 2.3$ \\
\hline Height $(\mathrm{cm})$ & $177.0 \pm 1.4$ & $173.0 \pm 1.3$ & $173.0 \pm 1.6$ & $174 \pm 0.9$ \\
\hline BMI $\left(\mathrm{kg} / \mathrm{m}^{2}\right)$ & $26.2 \pm 1.1$ & $23.5 \pm 0.8$ & $23.5 \pm 1.3$ & $24.6 \pm 0.5$ \\
\hline Physical Inactivity & $8(57.1)$ & $8(57.1)$ & $8(61.5)$ & $24(58.5)$ \\
\hline \multicolumn{5}{|l|}{ Smoking } \\
\hline Smoker & $1(7.1)$ & $1(7.1)$ & $1(7.7)$ & $3(7.3)$ \\
\hline Non-smoker & $13(92.9)$ & $13(92.9)$ & $12(92.3)$ & $38(92.7)$ \\
\hline \multicolumn{5}{|c|}{ Alcohol Consumption } \\
\hline Drinker & $8(57.1)$ & $11(78.6)$ & $5(38.5)$ & $24(58.5)$ \\
\hline Non-drinker & $6(42.9)$ & $3(21.4)$ & $8(61.5)$ & $17(41.5)$ \\
\hline
\end{tabular}

TEAS = Transcutaneous electrical acupoint stimulation group; BMI = body mass index; SE = standard error of the mean. 

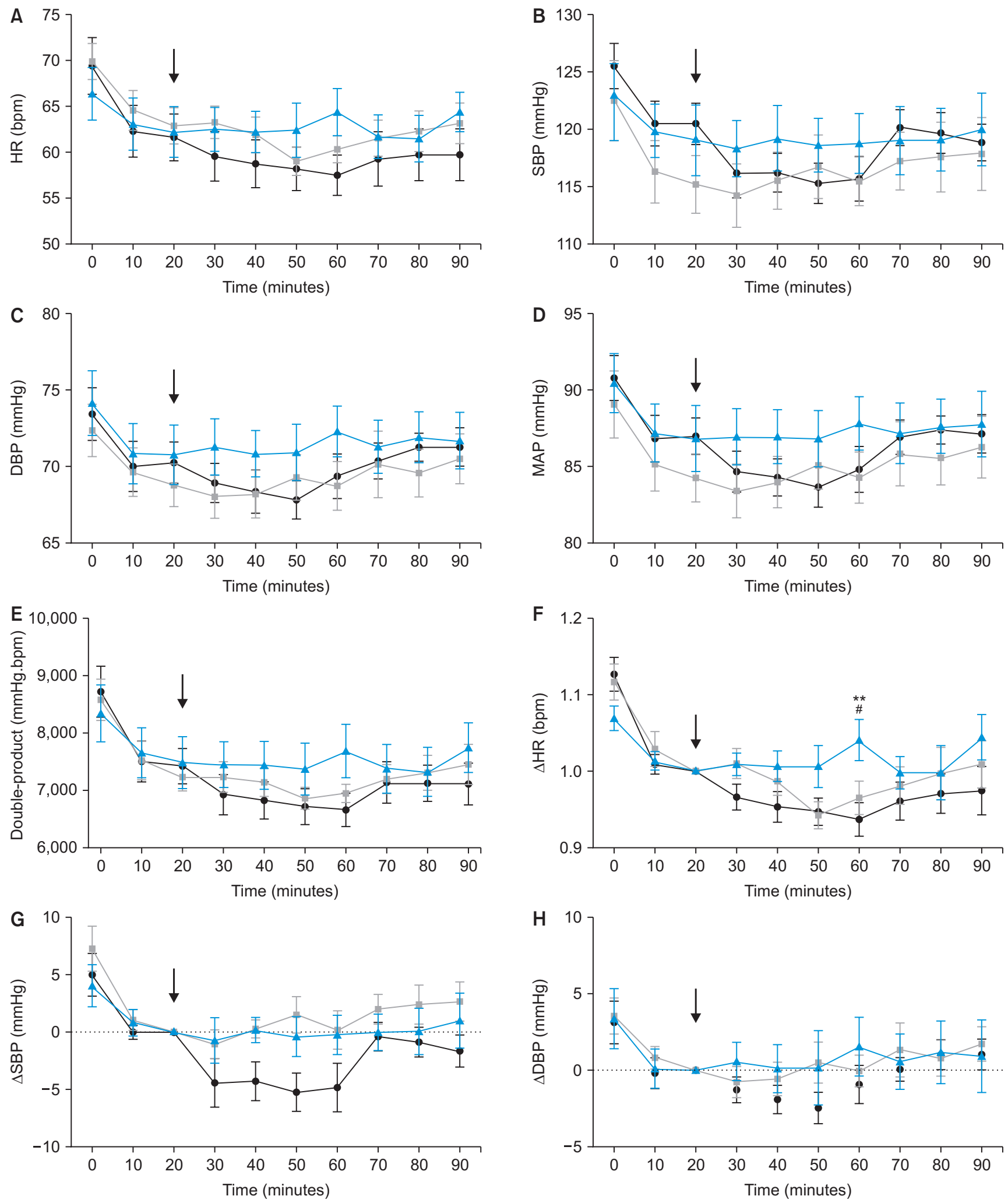

Fig. 2. Heart Rate, Blood Pressure and Double Product records and variations. ( $)$ Control, ( $\square$ ) Placebo and ( $\mathbf{\Delta})$ TEAS group. The black arrow marks the beginning of the 40-minute intervention period. (A) Heart Rate (HR) record; (B) Systolic Blood Pressure (SBP) record; (C) Diastolic Blood Pressure (DBP) record; (D) Mean Arterial Pressure (MAP) record; (E) Double Product record; $(\mathrm{F})$ Heart Rate variation $(\triangle \mathrm{HR})$; $(\mathrm{G}) \mathrm{SBP}$ variation $(\triangle \mathrm{SBP})$; $(\mathrm{H}) \mathrm{DBP}$ variation $(\triangle \mathrm{DBP})$; (I) MAP variation $(\triangle M A P)$; $(\mathrm{J})$ Double Product variation. Two-way ANOVA statistical test with Bonferroni post-test. Significance level set at $p<0.05$. ${ }^{* *} p<0.01$ (TEAS vs control group); $" p<0.05$ (TEAS vs placebo group). 


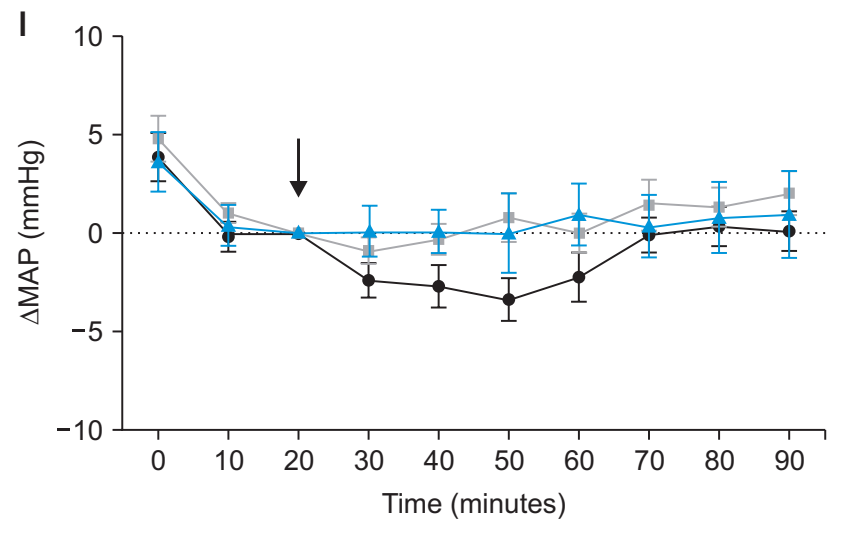

Fig. 2. Continued.

the baseline, intervention and recovery periods and used for oxidative stress analysis. Initially, the total protein concentration was determined using a protein assay kit (Pierce BCA Protein Assay Kit - ThermoFisher), for this, $10 \mu \mathrm{L}$ of saliva were mixed with $200 \mu \mathrm{L}$ of standard BCA reagent diluted in phosphate-saline buffer (PBS) into 96 wellsplates and incubated for 30 minutes for later reading of the absorbance (562 nm, with SpectraMax Plus 384, Molecular Devices). Then, the samples were diluted until achieving $800 \mu \mathrm{M}$, concentration used in the oxidative stress test. The 2'7'-dichlorofluorescein diacetate probe (DCFH-DA, Sigma, EUA) was used for quantifying ROS in saliva samples. Triplicates of saliva samples $(50 \mu \mathrm{L})$ were added with the same volume of DCFH-DA $40 \mu \mathrm{M}$ in 96 wells-plates and incubated for 20 minutes at $37^{\circ} \mathrm{C}$, for later fluorescence analysis using excitation $\lambda=488 \mathrm{~nm}$ and emission $=525 \mathrm{~nm}$ (SpectraMax Plus 384, Molecular Devices). Values are expressed in units of fluorescence (UF).

\section{Ethical considerations}

The study was conducted in accordance with the Helsinki Declaration of 1975, as revised in 2013. The ethics committee of the Federal University of the State of Rio de Janeiro (CAAE: 47780515.3.0000.5285) approved the study, and all volunteers signed an informed consent form before the beginning of the protocol. The study was registered in the Brazilian Clinical Trial Registry (ReBEC identifier: RBR-43byv6).

\section{Data analysis}

All results are expressed as mean \pm standard error of the mean (SE). Two-way ANOVA with Bonferroni posttest was used for comparing longitudinal effects of TEAS (Prism 7, GraphPad software, USA). $p<0.05$ was considered significant.

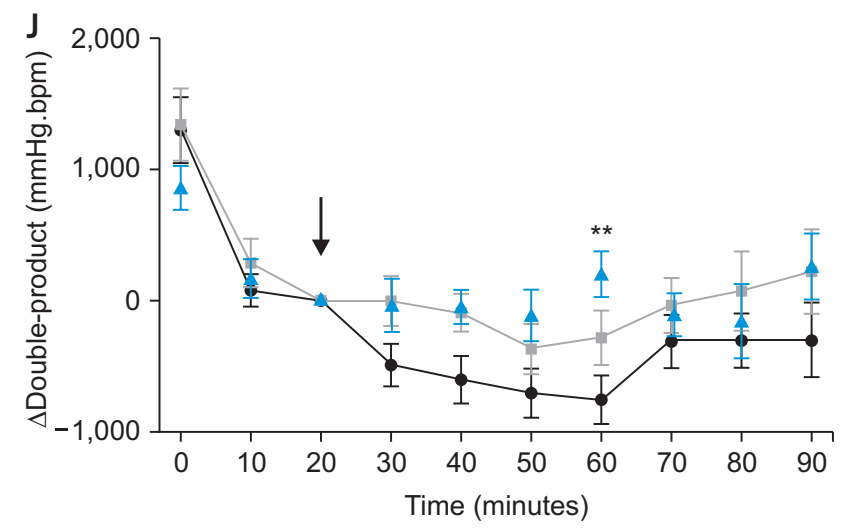

\section{RESULTS}

From January 2016 to March 2017, 96 patients were recruited for the study. From these, 47 accepted to participate, being assessed for eligibility. After initial assessment, 6 volunteers were excluded, being 41 randomized into one of the three experimental groups (Fig. 1).

The baseline sociodemographic characteristics of study participants are depicted in Table 1 . The mean age was approximately 21 years old and BMI was $24.6 \pm 0.5 \mathrm{~kg} /$ $\mathrm{m}^{2}$. The majority of participants self-reported as physically inactive (57.1\%), non-smoker (92.9\%) and regular drinkers (57.1\%).

Fig. 2 presents crude means and changes for the hemodynamic variables along the experimental protocol. There was no difference in HR, SBP, DBP, MAP or DP between experimental groups (Fig. 2A-E, respectively). However, at the end of the intervention period, TEAS significantly increased $\Delta$ HR compared to both control $(p=0.002)$ and placebo $(p$ $=0.043$ ) groups (Fig. $2 \mathrm{~F}$ ) and also $\Delta \mathrm{DP}$ compared to control $(p=0.003)$ group (Fig. 2J). No effects on $\triangle \mathrm{SBP}, \triangle \mathrm{DBP}$ and $\triangle \mathrm{MAP}$ were observed (Fig. 2G-I, respectively).

TEAS did not affect the absolute power of the frequency domain or time domain HRV variables along the experimental protocol (Table 2). However, during the intervention, TEAS significantly increased the normalized LF component compared to control $(p=0.015)$ and placebo $(p=0.018)$ groups (Fig. 3A). Also, TEAS reduced normalized HF component compared to control $(p=0.016)$ and placebo $(p=$ 0.019 ) groups (Fig. 3B). Moreover, compared to control and placebo groups, the sympathovagal index was increased by TEAS during the intervention period $(p=0.005$ and $p=0.007$, respectively) (Fig. 3C). Representatives power spectrum of HRV, obtained from a volunteer of the TEAS group, illustrate the effect of TEAS on HRV at baseline (Fig. 3D), intervention (Fig. 3E) and recovery (Fig. 3F) periods.

Finally, compared to control and placebo groups, TEAS did 
Table 2. Effects of TEAS on HRV and ROS

\begin{tabular}{|c|c|c|c|c|}
\hline & $\begin{array}{c}\text { Control }(N=14) \\
\text { Mean } \pm S E\end{array}$ & $\begin{array}{c}\text { Placebo }(\mathrm{N}=14) \\
\text { Mean } \pm \text { SE }\end{array}$ & $\begin{array}{l}\text { TEAS }(\mathrm{N}=13) \\
\text { Mean } \pm \mathrm{SE}\end{array}$ & $p$-value \\
\hline \multicolumn{5}{|l|}{ Mean RR (ms) } \\
\hline Baseline & $988.8 \pm 48.0$ & $953.8 \pm 24.0$ & $978.9 \pm 49.5$ & 0.129 \\
\hline Intervention & $1057.9 \pm 42.9$ & $993.6 \pm 26.4$ & $969.1 \pm 44.1$ & \\
\hline Recovery & $1030.0 \pm 44.7$ & $973.2 \pm 29.0$ & $963.2 \pm 38.0$ & \\
\hline \multicolumn{5}{|l|}{ SDNN (ms) } \\
\hline Baseline & $67.5 \pm 6.9$ & $69.3 \pm 7.7$ & $68.9 \pm 7.0$ & 0.452 \\
\hline Intervention & $79.4 \pm 5.6$ & $88.0 \pm 8.6$ & $90.3 \pm 11.0$ & \\
\hline Recovery & $76.3 \pm 7.0$ & $92.4 \pm 6.7$ & $89.6 \pm 10.4$ & \\
\hline \multicolumn{5}{|l|}{ RMSSD (ms) } \\
\hline Baseline & $64.0 \pm 8.4$ & $68.1 \pm 9.7$ & $62.6 \pm 9.1$ & 0.598 \\
\hline Intervention & $78.2 \pm 7.7$ & $80.9 \pm 9.4$ & $71.6 \pm 11.3$ & \\
\hline Recovery & $65.8 \pm 8.2$ & $78.6 \pm 12.4$ & $69.1 \pm 9.5$ & \\
\hline \multicolumn{5}{|l|}{ NN50 (count) } \\
\hline Baseline & $174.6 \pm 27.6$ & $211.3 \pm 37.8$ & $182.5 \pm 29.3$ & 0.409 \\
\hline Intervention & $228.0 \pm 25.8$ & $237.3 \pm 31.7$ & $196.7 \pm 30.9$ & \\
\hline Recovery & $168.9 \pm 30.8$ & $234.0 \pm 31.7$ & $203.5 \pm 32.4$ & \\
\hline \multicolumn{5}{|l|}{ pNN50 (\%) } \\
\hline Baseline & $37.5 \pm 5.9$ & $37.8 \pm 6.6$ & $34.7 \pm 6.0$ & 0.135 \\
\hline Intervention & $46.7 \pm 5.0$ & $43.7 \pm 5.6$ & $36.2 \pm 6.1$ & \\
\hline Recovery & $37.4 \pm 6.1$ & $41.6 \pm 5.8$ & $36.5 \pm 5.7$ & \\
\hline \multicolumn{5}{|l|}{$\operatorname{VLF}\left(\mathrm{ms}^{2}\right)$} \\
\hline Baseline & $1590.8 \pm 359.4$ & $1719.1 \pm 571.6$ & $1581.5 \pm 201.2$ & 0.187 \\
\hline Intervention & $1768.4 \pm 324.8$ & $2326.2 \pm 513.6$ & $4007.7 \pm 1194.1$ & \\
\hline Recovery & $2171.5 \pm 646.6$ & $2983.6 \pm 316.1$ & $4182.5 \pm 982.8$ & \\
\hline \multicolumn{5}{|l|}{$\operatorname{LF}\left(\mathrm{ms}^{2}\right)$} \\
\hline Baseline & $1399.5 \pm 351.0$ & $1435.9 \pm 317.4$ & $1361.0 \pm 329.7$ & 0.158 \\
\hline Intervention & $1856.1 \pm 280.3$ & $2362.1 \pm 438.0$ & $3091.8 \pm 656.7$ & \\
\hline Recovery & $1623.0 \pm 271.7$ & $2415.1 \pm 446.5$ & $2224.9 \pm 473.3$ & \\
\hline \multicolumn{5}{|l|}{$\mathrm{HF}\left(\mathrm{ms}^{2}\right)$} \\
\hline Baseline & $1710.0 \pm 459.2$ & $2254.6 \pm 517.0$ & $1825.4 \pm 572.7$ & 0.798 \\
\hline Intervention & $2098.1 \pm 318.8$ & $2722.2 \pm 662.6$ & $2067.5 \pm 656.5$ & \\
\hline Recovery & $1568.1 \pm 304.0$ & $2705.3 \pm 916.7$ & $2121.2 \pm 591.8$ & \\
\hline \multicolumn{5}{|l|}{ ROS (UF) } \\
\hline Baseline & $292.1 \pm 77.2$ & $340.7 \pm 88.5$ & $486.3 \pm 258.3$ & 0.511 \\
\hline Intervention & $314.0 \pm 90.0$ & $458.2 \pm 141.4$ & $482.1 \pm 111.9$ & \\
\hline Recovery & $207.3 \pm 54.6$ & $405.9 \pm 119.6$ & $411.5 \pm 100.9$ & \\
\hline
\end{tabular}

TEAS = Transcutaneous electrical acupoint stimulation group; VLF = very low frequency component; LF = low frequency component; $\mathrm{HF}$ = high frequency component; SDNN = standard deviation of normal RR interval; RMSSD = root-mean-square of successive differences; $N N 50=$ number of consecutive normal RR interval differences greater than 50 ms; pNN50 = percentage of normal RR intervals that differed by more than $50 \mathrm{~ms}$ from the adjacent interval; ROS = reactive oxygen species. Statistical analysis using Two-way ANOVA with Bonferroni, $p<0.05$ considered statistically significant.

not affect ROS along the experimental protocol (Table 2).

\section{DISCUSSION}

The main finding of the present study was that TEAS, realized at PC5 and PC6 acupoints of male healthy subjects, increased sympathetic and reduced parasympathetic autonomic tonus, acutely increasing sympathovagal balance during the intervention, which consistently resulted in HR, SBP and DP increase, findings that ceased after the recovery period. Despite the observed HRV and hemodynamic effects, TEAS did not affect oxidative stress.

Several effects of PC5 and PC6 acupoints stimulation have been previously reported, demonstrating that acupuncture, EA and TEAS can induce cardiovascular effects such as the improvement of endothelial dysfunction in hypertensive 

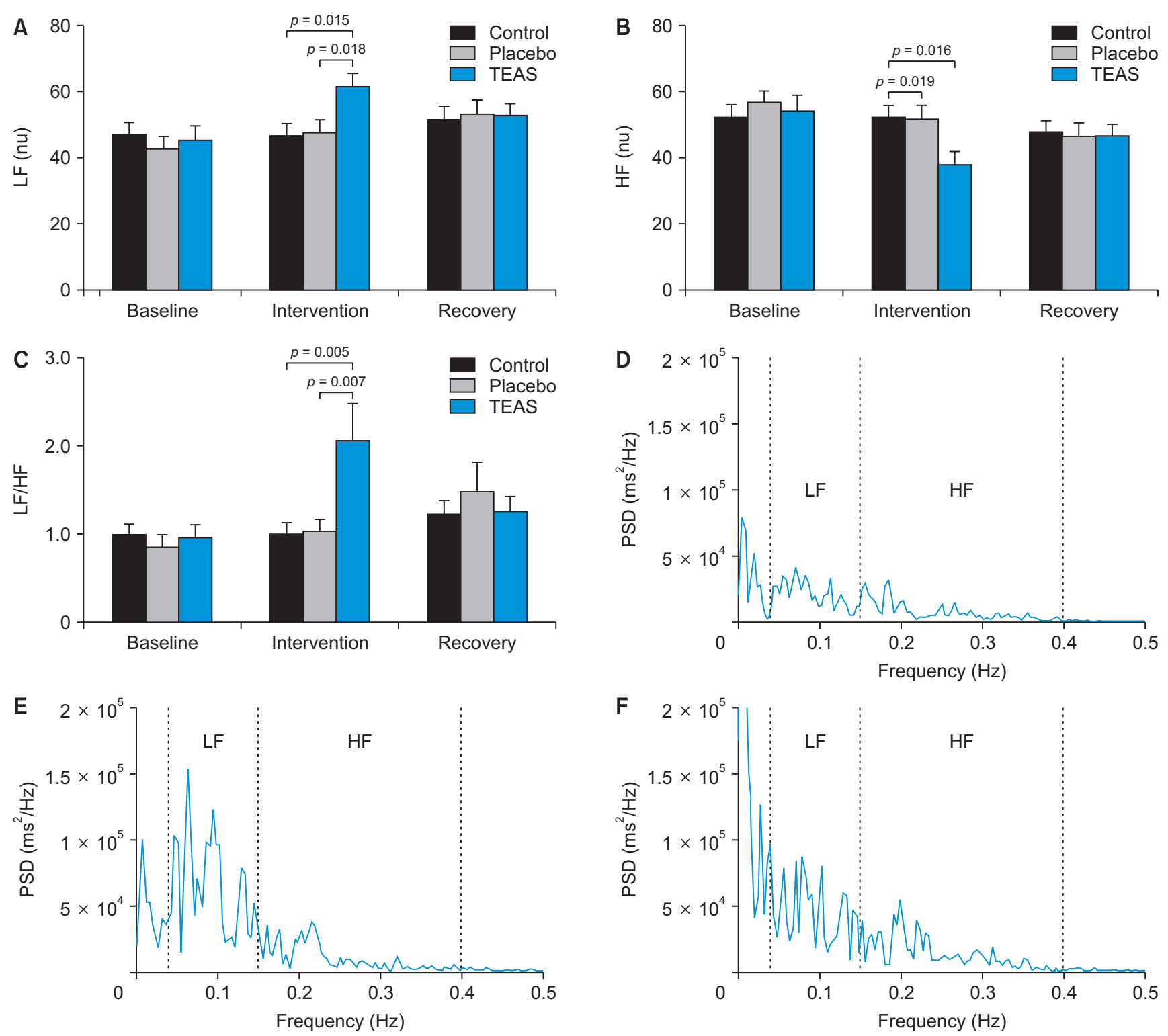

Fig. 3. Effects of TEAS on sympathovagal balance. (A) Low frequency component (LF) in normalized unit (nu); (B) high frequency component; (HF) in normalized unit (nu); (C) Sympathovagal index (LF/HF) at baseline, intervention and recovery period, respectively. Representative power spectrum density (PSD) of HRV, obtained from a volunteer of the TEAS group, illustrating the effect of TEAS on HRV at the end of baseline (D), intervention (E) and recovery (F) periods. Two-way ANOVA statistical test with Bonferroni post-test. Significance level set at $p<0.05$.

patients [20], reduction of myocardial ischemia injury and its severity [9], reduced arrhythmias incidence in human and animal models with ischemic injury [21] and increase in exercise tolerance in patients with heart failure [22].

Acupuncture and EA at PC6 acupoint have been reported to cause hemodynamics benefits by improving myocardial ischemic dysfunction through a pressure depressing response and inducing a pressure response in the recovery of hemorrhagic hypotension [23]. In pregnant women undergoing Caesarean section, an increase in the pressure response in hypotension after anesthesia was demonstrated due to
TEAS at PC5 and PC6 acupoint [24]. However, in the present study the absent response of crude heart rate and blood pressure values observed during TEAS at PC5 and PC6 acupoints could be explained by the integrity of ANS control under HR and blood pressure of healthy subjects, which could lead to physiological adjustments in vascular tonus in order to maintain mean blood pressure under normal levels. Hemodynamic effects were observed only when the variations of HR and DP were analyzed at the end of the baseline period, evidencing a small but significant increase of these variables. On the other hand, when TEAS at PC5 and PC6 acupoints 
was applied in heart transplant recipients, known of having worse HR control due to ANS denervation and impairment after surgery, crude SBP, DBP and MAP slightly increased but with a reduced HR after the end of electrostimulation period [18]. The increase in the DP variation (a non-invasive indicator of myocardial work) observed in the present study suggests a rise in oxygen consumption by myocardium during TEAS, usually explained by the increase in sympathetic activity $[25,26]$.

In fact, healthy individuals tend to have higher HRV compared with those with CVDs [27] due to the more active autonomic modulation, which allows a better HR regulation through physiological and environmental stimuli [28]. It was already described a decrease in HRV in hypertensive patients when compared to normotensive subjects, with a significant reduction in SDNN and pNN50 indexes, probably related to sympathetic hyperactivity [29]. It was also reported a reduction in HRV after acute myocardial infarction [30] and a reduction in vagal activity and cardiac function mediated by parasympathetic nervous system in patients with coronary arterial disease and myocardial infarctation [31]. In the present study, TEAS at PC5 and PC6 acupoints transitorily increased sympathetic and reduced parasympathetic activity, resulting in an increase of sympathovagal balance during stimulation. Such findings may provide a promising clinical applicability of this technique, since a single session of TEAS seems to act similarly to an aerobic exercise session, leading to an acute increase in sympathetic activity and a reduction in parasympathetic activity [32,33]. However, these effects on autonomic modulation, with a predominance of sympathetic activity that ceased during the recovery period, demonstrates an acute and transitory effect of TEAS on hemodynamics of healthy subjects, leading to a question: could the TEAS present similar temporal summation as exercise? Similarly, it was already demonstrated that the stimulation of the PC6 acupoint led to sympathetic-excitatory effect such as increased cardiac output, vasomotor tonus and cardiac function [23,24]. Also, increased sympathetic activity and sympathovagal balance were demonstrated during the TEAS at PC5 and PC6 acupoints in heart transplant recipients [18]. Nevertheless, despite the evidences regarding TEAS effect on autonomic function, the mechanisms underlining those modulation is still unknown, being necessary some mechanistic studies relating TEAS, neuroimages and autonomic function, since acupuncture affects a different brain regions and their associated functions, and ANS regulates several vital functions by the reflexes arcs that integrates peripheral sensors, the central nervous system and effector organs [34].

Our study was the first evaluating cardioprotective effects of TEAS at PC5 and PC6 acupoints of healthy subjects through oxidative stress quantification [16], an innovative approach since ROS generation is strongly related to the development of CVDs. Despite the HRV and hemodynamic effects demonstrated in the present study, TEAS exerted no effect on oxidative stress of healthy individuals. However, acupuncture stimulation techniques have already shown effectiveness in reducing oxidative stress in animal models. In rats submitted to testicular torsion and detorsion, the electroacupuncture applied bilaterally to the acupoint Gulai (S29), in a single session, reduced ROS generation, with the increase of the testicular and serum glutathione (GSH) concentration and decreased activity of testicular myeloperoxidase (MPO), but with an enhance of Malondialdehyde (MDA) [14]. Also, it was demonstrated the effectiveness of acupuncture in reducing oxidative stress after ischemia-reperfusion injury, improving the action of antioxidant enzymes and declining the formation of ROS [35]. Finally, rats subjected to ovarian damage by radiation obtained an increase in the levels of Glutathione Peroxidase (GSH-Px) and Superoxide dismutase 2 (SOD2) and a reduction in the concentration of MDA in the ovary after 2 weeks of TEAS sessions in acupoint Guanyuan (CV4) [15]. Interestingly, those beneficial findings of acupoint stimulation on oxidative stress were only previously observed in high ROS formation models, as chronic diseases, while the present study used self-reported healthy subjects without any disease associated with increase of ROS formation, and a single session of TEAS, that could impair the observation of the effects of TEAS on autonomic function, hemodynamics and ROS generation.

\section{LIMITATIONS}

The use of saliva samples, instead of blood samples, was chosen for the quantification of ROS because the rapid and non-invasive nature of the procedure, avoiding the need for repeated insertion of needles or use of venous access for serial blood collection. However, this may represent an important study limitation since changes in ROS in saliva may occur more slowly than in blood and tissues, making it difficult to observe acute variations. Among the three groups, TEAS group was the only group that received electrical stimulation, potentially affecting the HRV changes observed in this study, the lack of a placebo group with electrical stimulation of nonacupoints regions may be considered in future studies.

\section{CONCLUSIONS}

A single-session of TEAS at PC5 and PC6 acupoints acutely increased hemodynamics and autonomic balance of healthy individuals, increasing sympathetic and reducing parasympathetic tonus during the intervention, that ceased soon after the intervention. Despite of the observed effects 
on ANS, the use of TEAS at PC5 and PC6 acupoints as a cardioprotective strategy remains uncertain since it exerted no acute effect on oxidative stress, that is strongly associated with development of chronic inflammatory diseases, including CVDs. Longitudinal studies are necessary for investigating the use of TEAS at PC5 and PC6 acupoints as cardioprotective strategy against CVDs.

\section{ACKNOWLEDGEMENTS}

Thanks to the researchers from the Department of Physiological Sciences from the Federal University of the State of Rio de Janeiro.

\section{CONFLICT OF INTEREST}

The authors declare no conflict of interest.

\section{ORCID}

Fernando Gomes de Jesus, https://orcid.org/0000-0001-8129-1943

Alice Pereira Duque,

https://orcid.org/0000-0003-0325-9424

Carole Sant'ana Massolar, https://orcid.org/0000-0002-6840-4090

Giselle Pinto de Faria Lopes, https://orcid.org/0000-0001-9502-2314

Ana Carolina de Azevedo Carvalho, https://orcid.org/0000-0002-5771-3621

Mauro Felippe Felix Mediano, https://orcid.org/0000-0001-6369-3631

Luiz Fernando Rodrigues Junior, https://orcid.org/0000-0001-7007-7431

\section{REFERENCES}

1. Laslett LJ, Alagona P Jr, Clark BA 3rd, Drozda JP Jr, Saldivar F, Wilson SR, et al. The worldwide environment of cardiovascular disease: prevalence, diagnosis, therapy, and policy issues: a report from the American College of Cardiology. J Am Coll Cardiol 2012;60(25 Suppl):S1-49.

2. Bowry AD, Lewey J, Dugani SB, Choudhry NK. The burden of cardiovascular disease in low- and middle-income countries: epidemiology and management. Can J Cardiol 2015;31:1151-9.

3. Mendis S, Puska P, Norrving B. Global Atlas on Cardiovascular Disease Prevention and Control. Geneva: World Health Organization, 2011.

4. Gawryszewski VP, Souza Mde F. Mortality due to cardiovascular diseases in the Americas by region, 2000-2009. Sao Paulo Med J. 2014;132:105-10.
5. Mostarda C, Wichi R, Sanches IC, Rodrigues B, De Angelis $\mathrm{K}$, Irigoyen MC. [Hypertension and autonomic modulation in olders: role of exercise training]. Rev Bras Hipertens 2009;16:5560. Portuguese.

6. Kim HG, Cheon EJ, Bai DS, Lee YH, Koo BH. Stress and heart rate variability: a meta-analysis and review of the literature. Psychiatry Investig 2018;15:235-45.

7. Syuu Y, Matsubara H, Kiyooka T, Hosogi S, Mohri S, Araki J, Ohe T, et al. Cardiovascular beneficial effects of electroacupuncture at Neiguan (PC-6) acupoint in anesthetized openchest dog. Jpn J Physiol 2001;51:231-8.

8. Sahmeddini MA, Eghbal MH, Khosravi MB, Ghaffaripour S, Janatmakan F, Shokrizade S. Electro-acupuncture stimulation at acupoints reduced the severity of hypotension during anesthesia in patients undergoing liver transplantation. J Acupunct Meridian Stud 2012;5:11-4.

9. Meng J. The effects of acupuncture in treatment of coronary heart diseases. J Tradit Chin Med 2004;24:16-9.

10. Ma D, Han JS, Diao QH, Deng GF, Ping XJ, Jin WJ, et al. Transcutaneous electrical acupoint stimulation for the treatment of withdrawal syndrome in heroin addicts. Pain Med 2015;16:83948.

11. Ni X, Xie Y, Wang Q, Zhong H, Chen M, Wang F, et al. Cardioprotective effect of transcutaneous electric acupoint stimulation in the pediatric cardiac patients: a randomized controlled clinical trial. Paediatr Anaesth 2012;22:805-11.

12. Chien LW, Lin MH, Chung HY, Liu CF. Transcutaneous electrical stimulation of acupoints changes body composition and heart rate variability in postmenopausal women with obesity. Evid Based Complement Alternat Med 2011;2011: 862121.

13. Du SQ, Wang XR, Zhu W, Ye Y, Yang JW, Ma SM, et al. Acupuncture inhibits TXNIP-associated oxidative stress and inflammation to attenuate cognitive impairment in vascular dementia rats. CNS Neurosci Ther 2018;24:39-46.

14. Acioli PC, Albuquerque Ade O, Guimarães IB, Araujo RW, Vasconcelos PR, Guimarães SB. Protective effects of abdominal electroacupuncture on oxidative stress and inflammation due to testis torsion/detorsion in rats. Acta Cir Bras 2014;29:450-6.

15. Tan R, He Y, Zhang S, Pu D, Wu J. Effect of transcutaneous electrical acupoint stimulation on protecting against radiotherapy- induced ovarian damage in mice. J Ovarian Res 2019; 12:65.

16. Rubio MC, González PM, Ramos C, Lewin PG, Friedman SM, Puntarulo S, et al. Oxidative stress assessed in saliva from patients with acute myocardial infarction. A preliminary study. Acta Odontol Latinoam 2013;26:116-20.

17. Yang L, Yang J, Wang Q, Chen M, Lu Z, Chen S, et al. Cardioprotective effects of electroacupuncture pretreatment on patients undergoing heart valve replacement surgery: a randomized controlled trial. Ann Thorac Surg 2010;89:781-6. 
18. Moreira BR, Duque AP, Massolar CS, de Lima Pimentel R, Mediano MFF, Guimarães TCF, et al. Transcutaneous electrical stimulation of PC5 and PC6 acupoints modulates autonomic balance in heart transplant patients: a pilot study. J Acupunct Meridian Stud 2019;12:84-9.

19. WHO. WHO Standard Acupuncture Point Locations in the Western Pacific Region. Manila: Werstern Pacific Region Office, 2008.

20. Park JM, Shin AS, Park SU, Sohn IS, Jung WS, Moon SK. The acute effect of acupuncture on endothelial dysfunction in patients with hypertension: a pilot, randomized, double-blind, placebo-controlled crossover trial. J Altern Complement Med 2010;16:883-8.

21. Longhurst JC. Electroacupuncture treatment of arrhythmias in myocardial ischemia. Am J Physiol Heart Circ Physiol 2007;292: H2032-4.

22. Kristen AV, Schuhmacher B, Strych K, Lossnitzer D, Friederich HC, Hilbel T, et al. Acupuncture improves exercise tolerance of patients with heart failure: a placebo-controlled pilot study. Heart 2010;96:1396-400.

23. Syuu Y, Matsubara H, Hosogi S, Suga H. Pressor effect of electroacupuncture on hemorrhagic hypotension. Am J Physiol Regul Integr Comp Physiol 2003;285:R1446-52.

24. Arai YC, Kato N, Matsura M, Ito H, Kandatsu N, Kurokawa S, et al. Transcutaneous electrical nerve stimulation at the PC-5 and PC-6 acupoints reduced the severity of hypotension after spinal anaesthesia in patients undergoing Caesarean section. $\mathrm{Br}$ J Anaesth 2008;100:78-81.

25. Miranda H, Simão R, Lemos A, Dantas BHA, Baptista LA, Novaes J. [Analysis on the cardiac rate, blood pressure and doubled-product in different body positions in resisted exercises]. Rev Bras Med Esporte 2005;11:295-8. Portuguese.

26. Maior AS, Gonçalves R, Marocolo M. [Acute response of blood pressure, heart rate and dual output after an electro-stimulation session with strength training exercises]. Rev SOCERJ 2007;20: 28-34. Portuguese.

27. Leite A, Silva ME, Rocha AP. [Heart rate variability analysis in healthy subjects, patients suffering from congestive heart failure and heart transplanted patients]. Motricidade 2013;9:54-63. Portuguese.

28. Zhang J. Effect of age and sex on heart rate variability in healthy subjects. J Manipulative Physiol Ther 2007;30:374-9.

29. Menezes Ada S Jr, Moreira HG, Daher MT. Analysis of heart rate variability in hypertensive patients before and after treatment with angiotensin II-converting enzyme inhibitors. Arq Bras Cardiol 2004;83:169-72; 165-8.

30. Pecyna MB. The level of intelligence and heart rate variability in men after myocardial infarction. J Physiol Pharmacol 2006;57 Suppl 4:283-7.

31. Tsou MT, Huang CH, Chiu JH. Electroacupuncture on PC6 (Neiguan) attenuates ischemia/reperfusion injury in rat hearts. Am J Chin Med 2004;32:951-65.

32. Abad CCC, Silva RS, Mostarda C, Silva ICM, Irigoyen MC. [Effect of resistance and aerobic exercise on the autonomic control and hemodynamic variables in health young individuals]. Rev Bras Educ Fis Esporte 2010;24:535-44. Portuguese.

33. Almeida MB, Araújo CGS. [Efeitos do treinamento aeróbico sobre a freqüência cardíaca]. Rev Bras Med Esporte 2003;9:104112. Portuguese.

34. Anderson B, Nielsen A, McKee D, Jeffres A, Kligler B. Acupuncture and heart rate variability: a systems level approach to understanding mechanism. Explore (NY) 2012;8:99-106.

35. Chen $\mathrm{CH}$, Hsieh CL. Effect of acupuncture on oxidative stress induced by cerebral ischemia-reperfusion injury. Antioxidants (Basel) 2020;9:248. 